\title{
Hamiltonicity of the Complete Double Vertex Graph of some Join Graphs
}

\author{
Luis Manuel Rivera, ${ }^{\circledR}$ Ana Laura Trujillo-Negrete ${ }^{\circledR}$
}

\begin{abstract}
The complete double vertex graph $M_{2}(G)$ of $G$ is defined as the graph whose vertices are the 2-multisubsets of $V(G)$, and two of such vertices are adjacent in $M_{2}(G)$ if their symmetric difference (as multisets) is a pair of adjacent vertices in $G$. In this paper we exhibit an infinite family of graphs $G$ (containing Hamiltonian and non-Hamiltonian graphs) for which $M_{2}(G)$ are Hamiltonian.
\end{abstract}

\section{Introduction}

Throughout this paper, $G$ is a simple graph of order $n \geq 2$. The complete double vertex graph $M_{2}(G)$ of $G$ is the graph whose vertices are all the 2-multisubsets of $V(G)$ where two vertices are adjacent whenever their symmetric difference (as multisubsets) is a pair of adjacent vertices in $G$. See an example in Figure 1. This class of graphs can be seen as a generalization of the 2-token graph $F_{2}(G)$ of $G$, where the vertex set consists of all the 2-subsets of $V(G)$ and the adjacencies are defined in a similar

2000 AMS Subject Classification: 05C45, 05C76.

Keywords and Phrases: Complete Double Vertex Graphs, Hamiltonicity, Token graphs.

A. L. Trujillo-Negrete was partially supported by CONACYT (Mexico), grant 253261. 


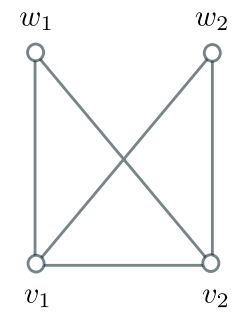

$F_{2,2}$

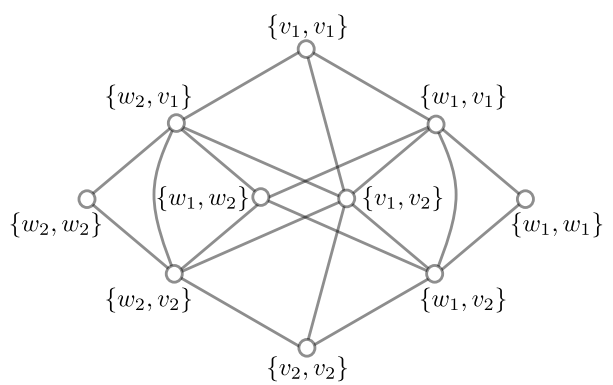

$M_{2}\left(F_{2,2}\right)$

Figure 1: Graph $F_{2,2}$ and its complete double vertex graph $M_{2}\left(F_{2,2}\right)$.

way. It is easy to show that $G$ and $F_{2}(G)$ are isomorphic to a subgraph of $M_{2}(G)$.

The 2-token graph is, indeed, a particular case of the $k$-token graphs. For a graph $G$ of order $n$ and an integer $k$ with $1 \leq k \leq n-1$, the $k$-token graph $F_{k}(G)$ of $G$ is the graph whose vertices are the $k$-subsets of $V(G)$, where two of such $k$-subsets are adjacent if their symmetric difference is a pair of adjacent vertices in $G$.

The properties of token graphs have been studied since 1991 by various authors and with different names, see, e.g., [1, 2, 3, 5, 9] and, in recent years, the study of its combinatorial properties and its connection with problems in other areas such as Coding Theory and Physics has increased, see, e.g., 44, 6, 8, 10, 11, 12, 14, 15. However, there are only few papers about complete double vertex graphs, which were implicitly introduced by Chartrand et al. [7]. As far as we know, the only parameters of complete double vertex graphs that have been studied are: connectivity, regularity, planarity, chromatic number, Hamiltonicity and Eulerianicity. These parameters were studied by Jacob, Goddard and Laskar [13] in 2007.

In this note we are interested in the Hamiltonicity of complete double vertex graphs. Jacob, Goddard and Laskar [13] showed that the Hamiltonicity of $G$ does not necessarily imply the Hamiltonicity of $M_{2}(G)$, for example if $n=4$ or $n \geq 6$, then $M_{2}\left(C_{n}\right)$ is not Hamiltonian. However, the 
authors also showed that if $G^{\prime}$ is the graph obtained from $C_{n}$ by adding an edge between two vertices at distance two, then $M_{2}\left(G^{\prime}\right)$ is Hamiltonian. To our knowledge, these are the only known results about the Hamiltonicity problem in this class of graphs.

In order to formulate our main result we need the following definitions. Given two disjoint graphs $G$ and $H$, the join graph $G+H$ of $G$ and $H$ is the graph whose vertex set is $V(G) \cup V(H)$ and its edge set is $E(G) \cup E(H) \cup$ $\{x y: x \in G$ and $y \in H\}$. The generalized fan graph, $F_{m, n}$, or simply the fan graph, is the join graph $F_{m, n}=E_{m}+P_{n}$, where $E_{m}$ denotes the empty graph on $m$ vertices $\left(E_{m}=\overline{K_{m}}\right)$ and $P_{n}$ denotes the path graph on $n$ vertices. In Figure 1 we show $F_{2,2}$.

The authors of this article studied the Hamiltonicity of $k$-token graphs of fan graphs $F_{1, n}$ [16]. In this note, we continue with this line of research but for the case of the complete double vertex graph of the generalized fan graphs $F_{m, n}$, for $m \geq 1$. Our main result is the following.

Theorem 1. The complete double vertex graph of $F_{m, n}$ is Hamiltonian if and only if $n \geq 2$ and $1 \leq m \leq 2(n-1)$.

A direct consequence of Theorem 1 is the following result.

Corollary 2. Let $G_{1}$ and $G_{2}$ be two graphs of order $m \geq 1$ and $n \geq 2$, respectively, such that $G_{2}$ has a Hamiltonian path. Let $G=G_{1}+G_{2}$. If $m \leq 2(n-1)$ then $M_{2}(G)$ is Hamiltonian.

Observe that Corollary 2 provides an infinite family of non-Hamiltonian graphs whose complete double vertex graphs are Hamiltonian.

\section{Proof of Theorem 1}

We introduce the following notation. Let $V\left(P_{n}\right):=\left\{v_{1}, \ldots, v_{n}\right\}$ and $V\left(E_{m}\right):=\left\{w_{1}, \ldots, w_{m}\right\}$, so $V\left(F_{m, n}\right)=\left\{v_{1}, \ldots, v_{n}, w_{1}, \ldots, w_{m}\right\}$. For a path $T=a_{1} a_{2} \ldots a_{l-1} a_{l}$, we will use $\overleftarrow{T}$ to denote the reverse path $a_{l} a_{l-1} \ldots a_{2} a_{1}$. As usual, for a positive integer $r$ let $[r]:=\{1,2, \ldots, r\}$. 
If $n=1$ and $m \geq 1$, then $\operatorname{deg}\left(\left\{w_{i}, w_{i}\right\}\right)=1$ for any $i \in[m]$, which implies that $M_{2}\left(F_{m, n}\right)$ is not Hamiltonian, so we assume that $n \geq 2$.

The proof is by construction. We distinguish four cases: either $m=1$, $m=2(n-1), 1<m<2(n-1)$ or $m>2(n-1)$.

\section{- Case $m=1$}

For $1 \leq i \leq n$ let

$$
T_{i}:=\left\{v_{i}, w_{1}\right\}\left\{v_{i}, v_{i}\right\}\left\{v_{i}, v_{i+1}\right\}\left\{v_{i}, v_{i+2}\right\} \ldots\left\{v_{i}, v_{n}\right\} .
$$

Note the following

(a) $T_{i}$ is a path in $M_{2}\left(F_{m, n}\right)$, where $T_{n}=\left\{v_{n}, w_{1}\right\}\left\{v_{n}, v_{n}\right\}$;

(b) for any $i \in[n-1]$ the final vertex of $T_{i}$ is adjacent to the initial vertex of $\overleftarrow{T_{i+1}}$, so the paths $T_{i}$ and $\overleftarrow{T_{i+1}}$ can be concatenated as $T_{i} \overleftarrow{T_{i+1}}$

(c) for any $i \in[n-1]$ the final vertex of $\overleftarrow{T}_{i}$ is adjacent to the initial vertex of $T_{i+1}$, so the paths $\overleftarrow{T_{i}}$ and $T_{i+1}$ can be concatenated as $\overleftarrow{T_{i}} T_{i+1}$

Let

$$
C:= \begin{cases}T_{1} \overleftarrow{T_{2}} T_{3} \overleftarrow{T_{4}} \ldots T_{n-1} \overleftarrow{T_{n}}\left\{w_{1}, w_{1}\right\} & \text { if } n \text { is even } \\ \overleftarrow{T_{1}}\left\{w_{1}, w_{1}\right\} T_{2} \overleftarrow{T_{3}} T_{4} \ldots T_{n-1} \overleftarrow{T_{n}} & \text { if } n \text { is odd }\end{cases}
$$

We claim that $C$ is a Hamiltonian cycle in $M_{2}\left(F_{m, n}\right)$. Suppose that $n$ is even. Statements (b) and (c) together imply that the concatenation $T_{1} \overleftarrow{T_{2}} T_{3} \overleftarrow{T_{4}} \ldots T_{n-1} \overleftarrow{T_{n}}$ produces a walk in $M_{2}\left(F_{m, n}\right)$ Now, since $\left\{v_{n}, w_{1}\right\}$ (the final vertex of $\overleftarrow{T_{n}}$ ) is adjacent to $\left\{w_{1}, w_{1}\right\}$, and $\left\{w_{1}, w_{1}\right\}$ is adjacent to $\left\{v_{1}, w_{1}\right\}$ (the initial vertex of $T_{1}$ ), it follows that $C$ is a closed walk in $M_{2}\left(F_{m, n}\right)$. Finally, note that $\left\{V\left(T_{1}\right), \ldots, V\left(T_{n}\right),\left\{\left\{w_{1}, w_{1}\right\}\right\}\right\}$ is a partition of the vertex set of $M_{2}\left(F_{m, n}\right)$. Therefore, $C$ is a Hamiltonian cycle. By similar arguments, when $n$ is odd we have that $C$ is a Hamiltonian cycle in $M_{2}\left(F_{m, n}\right)$, as claimed. 
Let us note here that $\left\{v_{n}, w_{1}\right\}$ and $\left\{v_{n}, v_{n}\right\}$ are adjacent in $C$ (these two vertices correspond to the vertices of $\overleftarrow{T_{n}}$ ). This observation will be useful in the following two cases.

- Case $m=2(n-1)$.

Let $C$ be the cycle defined in the previous case, depending on the parity of $n$. Let

$$
P_{1}:=\left\{v_{n}, w_{1}\right\} \stackrel{C}{\rightarrow}\left\{v_{n}, v_{n}\right\}
$$

be the path obtained from $C$ by deleting the edge between $\left\{v_{n}, w_{1}\right\}$ and $\left\{v_{n}, v_{n}\right\}$. For $1<i \leq n-1$ let

$$
\begin{gathered}
P_{i}=\left\{w_{i}, v_{n}\right\}\left\{w_{i}, w_{i}\right\}\left\{w_{i}, v_{n-1}\right\}\left\{w_{i}, w_{1}\right\}\left\{w_{i}, v_{n-2}\right\}\left\{w_{i}, w_{i+(n-2)}\right\} \\
\left\{w_{i}, v_{n-3}\right\}\left\{w_{i}, w_{i+(n-3)}\right\} \ldots\left\{w_{i}, v_{2}\right\}\left\{w_{i}, w_{i+2}\right\}\left\{w_{i}, v_{1}\right\}\left\{w_{i}, w_{i+1}\right\} .
\end{gathered}
$$

Note that after the vertex $\left\{w_{i}, w_{1}\right\}$, the vertices of $P_{i}$ follow the pattern $\left\{w_{i}, v_{j}\right\}\left\{w_{i}, w_{i+j}\right\}$, from $j=n-2$ to 1 .

For $n \leq i \leq m$ let

$$
\begin{array}{r}
P_{i}=\left\{w_{i}, v_{n}\right\}\left\{w_{i}, w_{i}\right\}\left\{w_{i}, v_{n-1}\right\}\left\{w_{i}, w_{i+(n-1)}\right\}\left\{w_{i}, v_{n-2}\right\} \\
\left\{w_{i}, w_{i+(n-2)}\right\} \ldots\left\{w_{i}, v_{2}\right\}\left\{w_{i}, w_{i+2}\right\}\left\{w_{i}, v_{1}\right\}\left\{w_{i}, w_{i+1}\right\},
\end{array}
$$

where the sums are taken $\bmod m$ and with the convention that $m$ $(\bmod m)=m$. In this case, after $\left\{w_{i}, w_{i}\right\}$, the vertices in $P_{i}$ follow the pattern $\left\{w_{i}, v_{j}\right\}\left\{w_{i}, w_{i+j}\right\}$, from $j=n-1$ to 1 . We claim that

$$
P:=P_{1} P_{2} \ldots P_{m}\left\{v_{n}, w_{1}\right\}
$$

is a Hamiltonian cycle in $M_{2}\left(F_{m, n}\right)$.

Note the following

1. the final vertex of $P_{1}$ is $\left\{v_{n}, v_{n}\right\}$ while the initial vertex of $P_{2}$ is $\left\{w_{2}, v_{n}\right\}$, and these two vertices are adjacent in $M_{2}\left(F_{m, n}\right)$;

2. for $1<i<2(n-1)$, the final vertex of $P_{i}$ is $\left\{w_{i}, w_{i+1}\right\}$ while the initial vertex of $P_{i+1}$ is $\left\{w_{i+1}, v_{n}\right\}$, and also these two vertices are adjacent in $M_{2}\left(F_{m, n}\right)$; 
3. the final vertex of $P_{m}$ is $\left\{w_{m}, w_{1}\right\}$ while the initial vertex of $P_{1}$ is $\left\{v_{n}, w_{1}\right\}$, and these two vertices are adjacent in $M_{2}\left(F_{m, n}\right)$.

These three observations together imply that $P$ is a closed walk in $M_{2}\left(F_{m, n}\right)$. Now we show that $\left\{V\left(P_{1}\right), \ldots, V\left(P_{m}\right)\right\}$ is a partition of $V\left(M_{2}\left(F_{m, n}\right)\right)$. Notice that any vertex in $V\left(F_{m, n}\right)$ satisfies exactly one of the following conditions:

- The vertices of type $\left\{v_{i}, v_{j}\right\}$ belong to $P_{1}$ for any $i, j \in[n]$.

- The vertices of type $\left\{w_{i}, v_{j}\right\}$ belong to $P_{i}$ for any $i \in[m]$ and $j \in[n]$.

- The vertices of type $\left\{w_{i}, w_{i}\right\}$ belong to $P_{i}$ for any $i \in[m]$.

- Let $H:=\left\{\left\{w_{i}, w_{j}\right\} \in V\left(M_{2}\left(F_{m, n}\right)\right): i, j \in[m], i \neq j\right\}$. Note that

$$
|H|=\left(\begin{array}{c}
m \\
2
\end{array}\right)=\frac{m(m-1)}{2}=(n-1)(m-1),
$$

and furthermore, $\left|V\left(P_{\ell}\right) \cap H\right|=n-1$ for each $\ell \in\{2, \ldots, m\}$. Moreover, for a vertex $\left\{w_{i}, w_{j}\right\}$, with $i, j \in[m]$ and $i<j$, we claim that

$$
\left\{w_{i}, w_{j}\right\} \in \begin{cases}P_{j} & \text { if } i=1 \\ P_{i} & \text { if } i>1 \text { and }|j-i| \leq n-2 \\ P_{j} & \text { if } i>1 \text { and }|j-i|>n-2 .\end{cases}
$$

First suppose $i=1$. If $1<j \leq n-1$, then Equation (1) implies that $\left\{w_{1}, w_{j}\right\} \in P_{j}$, and if $n \leq j \leq m$, then $w_{1} \in$ $\left\{w_{j+1}, w_{j+2}, \ldots, w_{j+(n-1)}\right\}$ and hence Equation (2) implies that $\left\{w_{1}, w_{j}\right\} \in P_{j}$. Suppose now that $i>1$. If $j-i \leq n-2$, then $w_{j} \in\left\{w_{i+1}, w_{i+2}, \ldots, w_{i+(n-2)}\right\}$, and so by Equation (1) it follows that $\left\{w_{i}, w_{j}\right\} \in P_{i}$. If $j-i>n-2$ then $w_{i} \in$ $\left\{w_{j+1}, w_{j+2}, \ldots, w_{j+(n-1)}\right\}$, and so by Equation (2) we have that $\left\{w_{i}, w_{j}\right\} \in P_{j}$.

Thus, $P$ is a Hamiltonian cycle in $M_{2}\left(F_{m, n}\right)$. 
- Case $1<m<2(n-1)$.

We consider again the paths $P_{1}, \ldots, P_{m}$ defined in the previous case with a slight modification:

$-P_{1}^{\prime}=P_{1}$

- for $i \in\{2, \ldots, m-1\}$, let $P_{i}^{\prime}$ be the path obtained from $P_{i}$ by deleting the vertices of type $\left\{w_{i}, w_{j}\right\}$, for each $j>m$;

- let $P_{m}^{\prime}$ be the path obtained from $P_{m}$ by first interchanging $\left\{w_{m}, w_{m+1}\right\}$ and $\left\{w_{m}, w_{1}\right\}$ from their current positions in $P_{m}$, and then deleting the vertices of type $\left\{w_{m}, w_{j}\right\}$, for every $j>$ $m$.

We have that $P_{1}^{\prime}, \ldots, P_{m}^{\prime}$ are, indeed, disjoint paths in $M_{2}\left(F_{m, n}\right)$, and that $P_{i}^{\prime}$ has the same initial and final vertices as $P_{i}$ for $i \neq m$, so the concatenation

$$
P^{\prime}:=P_{1}^{\prime} \ldots P_{m}^{\prime}\left\{v_{n}, w_{1}\right\}
$$

corresponds to a cycle in $M_{2}\left(F_{m, n}\right)$. It is an easy exercise to show that $P^{\prime}$ is, in fact, a Hamiltonian cycle of $M_{2}\left(F_{m, n}\right)$.

- Case $m>2(n-1)$.

In this case we show that $M_{2}\left(F_{m, n}\right)$ is not Hamiltonian by using the following result stated in West's book [17].

Proposition (Prop. 7.2.3, [17]). If $G$ has a Hamiltonian cycle, then for each nonempty set $S \subseteq V(G)$, the graph $G-S$ has at most $|S|$ connected components.

So, for our purpose, we are going to exhibit a subset $S \subset V\left(M_{2}\left(F_{m, n}\right)\right)$ such that the graph $M_{2}\left(F_{m, n}\right)-S$ has at most $|S|$ connected components. Let

$$
\begin{aligned}
S & :=\left\{\left\{w_{i}, v_{j}\right\} \in V\left(M_{2}\left(F_{m, n}\right)\right): i \in[m] \text { and } j \in[n]\right\}, \\
T & :=\left\{\left\{w_{i}, w_{j}\right\} \in V\left(M_{2}\left(F_{m, n}\right)\right): i, j \in[m]\right\}, \\
R & :=\left\{\left\{v_{i}, v_{j}\right\} \in V\left(M_{2}\left(F_{m, n}\right)\right): i, j \in[n]\right\} .
\end{aligned}
$$


The set $\{S, T, R\}$ is a partition of $V\left(M_{2}\left(F_{m, n}\right)\right)$. Since any vertex in $T$ has its neighbors in $S$, the subgraph induced by $T$ in $M_{2}\left(F_{m, n}\right)-S$ is isomorphic to $\overline{K_{\left(\begin{array}{c}m+1 \\ 2\end{array}\right)}}$. On the other hand, the subgraph induced by $R$ is a component of $M_{2}\left(F_{m, n}\right)-S$. Since $|S|=m n,|T|=\left(\begin{array}{c}m+1 \\ 2\end{array}\right)$ and $m>2(n-1)$, we have that the number of connected components of $M_{2}\left(F_{m, n}\right)-S$ is

$$
|T|+1=\left(\begin{array}{c}
m+1 \\
2
\end{array}\right)+1>m n=|S| .
$$

This completes the proof of Theorem 1 .

\section{Open problems}

There is a natural generalization of the complete double vertex graphs: Let $G$ be a simple graph of $n$ vertices and let $k$ be an integer with $1 \leq k \leq$ $n-1$. The $k$-multiset graph $M_{k}(G)$ of $G$ is the graph whose vertices are the $k$-multisubsets of $V(G)$, with two vertices being adjacent in $M_{k}(G)$ if their symmetric difference (as multisets) is a pair of adjacent vertices in $G$. This generalization gives rise to several open problems.

Open problem 1. To establish whether or not the Hamiltonicity of $G$ implies the Hamiltonicity of $M_{k}(G)$ for $k>2$.

In this paper we provided an infinite family of non-Hamiltonian graphs with Hamiltonian complete double vertex graphs. A natural open problem is to study if this result can be generalized.

Open problem 2. Given an integer $k>2$, to establish whether or not there exist non-Hamiltonian graphs with Hamiltonian $k$-multiset graphs. 


\section{References}

[1] Y. Alavi, M. Behzad, P. Erdős and D. R. Lick, Double vertex graphs, J. Combin. Inform. System Sci., 16 (1991), no. 1, 37-50.

[2] Y. Alavi, D. R. Lick and J. Liu, Survey of double vertex graphs, Graphs Combin., 18 (2002), no. 4, 709-715.

[3] Y. Alavi, D. R. Lick and J. Liu, Hamiltonian cycles in double vertex graphs of bipartite graphs, Congr. Numerantium, 93 (1993), 65-72.

[4] H. de Alba, W. Carballosa, J. Leaños and L. M. Rivera, Independence and matching number of some token graphs, Australas. J. Combin. 76 (2020), no. 3, 387-403.

[5] K. Audenaert, C. Godsil, G. Royle and T. Rudolph, Symmetric squares of graphs, J. Comb. Theory B 97 (2007), 74-90.

[6] W. Carballosa, R. Fabila-Monroy, J. Leaños and L. M. Rivera, Regularity and planarity of token graphs, Discuss. Math. Graph Theory 37 (2017), no. 3, 573-586.

[7] G. Chartrand, D. Erwin, M. Raines and P. Zhang, Orientation distance graphs, J. Graph Theory, 36 (2001), no. 4, 230-241.

[8] J. Deepalakshmi, G. Marimuthu, A. Somasundaram and S. Arumugam, On the 2-token graph of a graph, AKCE Int. J. Graphs Comb. 17 (2020), 265-268, https://doi.org/10.1016/j.akcej.2019.05.002

[9] R. Fabila-Monroy, D. Flores-Peñaloza, C. Huemer, F. Hurtado, J. Urrutia and D. R. Wood, Token graphs, Graph Combinator. 28 (2012), no. 3, 365-380.

[10] R. Fabila-Monroy, J. Leaños and A. L. Trujillo-Negrete, On the Connectivity of Token Graphs of Trees, arXiv:2004.14526 (2020). 
[11] C. Fischbacher, A Schrödinger operator approach to higher spin XXZ systems on general graphs, Analytic Trends in Mathematical Physics, Contemp. Math., 741 (2020), 83-103.

[12] J. M. Gómez-Soto, J. Leaños, L. M. Ríos-Castro and L. M. Rivera, The packing number of the double vertex graph of the path graph, Discrete Appl. Math. 247 (2018), 327-340.

[13] J. Jacob, W. Goddard and R. Laskar, Double Vertex Graphs and Complete Double Vertex Graphs, Congr. Numer., 188 (2007), 161174.

[14] J. Leaños and A. L. Trujillo-Negrete, The connectivity of token graphs, Graphs Combin. 34 (2018), no. 4, 777-790.

[15] Y. Ouyang, Computing spectral bounds of the Heisenberg ferromagnet from geometric considerations, J. Math. Phys., 60 (2019), no. 7, 071901.

[16] L. M. Rivera and A. L. Trujillo-Negrete, Hamiltonicity of token graphs of fan graphs, Art Discr. Appl. Math., 1 (2018), \#P07.

[17] D. B. West, Introduction to Graph Theory, Second Edition. Pearson Education (Singapure), 2001.

Luis Manuel Rivera

Universidad Autónoma de Zacatecas, Mexico

Unidad Académica

Matemáticas

luismanuel.rivera@gmail.com
Ana Laura Trujillo-Negrete

Cinvestav, CDMX, Mexico

Departamento de Matemáticas

de ltrujillo@math.cinvestav.mx 